\title{
L'HUMAIN DE LA TERRE AU CEUR DE LA CRISE DE L'HUMANITÉ : UNE APPROCHE SYSTÉMIQUE DE LA SANTÉ DE LA TERRE À PARTIR DE LA RELATIVITÉ COGNITIVE ET SYSTÉMIQUE
}

\author{
Author(s) / Auteur(s) : \\ Richard Vitrac \\ Ingénieur ENSERG \\ richard.vitrac@gmail.com
}

\begin{abstract}
Résumé :
Cette contribution est dans le prolongement de l'exposé fait au congrès de Bruxelles en 2018 dont le titre était "la place de l'Europe dans la crise de l'humanité". La première partie de cette contribution faite par Richard Vitrac donnait une représentation cognitive et systémique de l'être humain défini à partir de la relativité cognitive et systémique (RCS).

La contribution actuelle se propose de montrer en quoi la RCS entraîne un changement de paradigme dans la représentation de l'être humain qui a des conséquences au niveau de la santé du corps social comme an niveau de la santé du corps humain. Avant la RCS l'être humain était identifié à son corps soumis à la causalité temporelle. Après le RCS il est identifié à sa conscience qui "expérimente" et gère son corps de chair.

Cela correspond à la formule de Teilhard de Chardin disant : "Nous ne sommes pas des êtres humains (sousentendu de chair) vivant une expérience spirituelle mais des êtres spirituels vivant une expérience humaine (sous-entendu de chair)!

Cette représentation nouvelle de l'humain justifie l'intuition de Teilhard de Chardin qui affirme que l'humanité est en train de passer du stade de la biosphère à celui de la noosphère où c'est la conscience qui est la racine de l'humain, ce n'est plus son corps.

La première partie met en évidence le fonctionnement de l'humain ancien (avant la RCS) soumis à la causalité temporelle. La deuxième partie montre comment la RCS met en évidence qu'effectivement la noosphère est déjà en train de naître dans les consciences. Cela modifiera radicalement la relation des humains avec l'idée qu'ils se font de Dieu ainsi qu'avec la nature, et en particulier avec la terre elle-même qui sera respectée, car l'homme saura qu'il est responsable du devenir de la maison terre.

Cela devrait permettre à l'humanité de sortir de la crise pour entrer dans la paix des consciences.
\end{abstract}

Keywords / Mots-clés :

crise, systémique, relativité cognitive et systémique, complexité, intrication, génétique, mémoire systémique, être humain

\section{INTRODUCTION}

La contribution est en deux parties. La première représente l'être humain "normal" qui est identifié à son corps et à ses sens qui viennent de son corps de chair soumis au temps. Majoritairement les scientifiques ont adhéré à cette représentation de l'humain "charnel" puisqu'ils ont postulé que tout dans l'univers est soumis à la causalité temporelle. Nous allons représenter cet humain charnel et montrer que son corps est effectivement soumis à la causalité temporelle mais non sa conscience et son esprit pensant. Dans cette première partie, nous donnerons une représentation systémique traditionnelle des interrelations entre les humains ou entre les humains et d'autres systèmes, animaux, plante, la terre, le système solaire.

Dans la deuxième partie nous donnerons une représentation de l'humain nouveau, celui qui prend en compte la présence de l'Observateur Relativiste présent en lui et Pilote de sa vie. Nous montrerons comment l'intrication systémique rend tous les êtres humains coresponsables du devenir de la terre.

La conclusion de cette contribution confortera l'intuition de Teilhard de Chardin affirmant que l'évolution de l'humanité la fait quitter le stade de la biosphère constituée des humains qui étaient 
identifiés à leurs corps biologiques, pour entrer dans la noosphère où nous serons identifiés à nos consciences qui naissent de la Conscience Absolue de l'Être qui est notre racine d'être.

\section{CHAPITRE 1.}

\section{LA REPRÉSENTATION DE L'HUMAIN ANCIEN "CHARNEL"}

\section{I.1. La représentation et le fonctionnement de l'humain "charnel"}

\section{I.1.1. Représentation de humain charnel, identifié à ses sens corporels}

La figure 1 représente un être humain assis sur la terre. Il est à l'instant présent $\mathrm{t}=0$ qui, dans le cas de la figure, correspond au zénith du soleil.

Il perçoit des corps avec son esprit identifié à ses 5 sens corporels. Comme la vitesse de transfert des signaux émis par les corps et perçus par l'humain n'est pas infinie, les corps perçus sont dans son passé.

S'il désire agir sur ces corps. Le résultat de son action qui a lieu à l'instant présent se

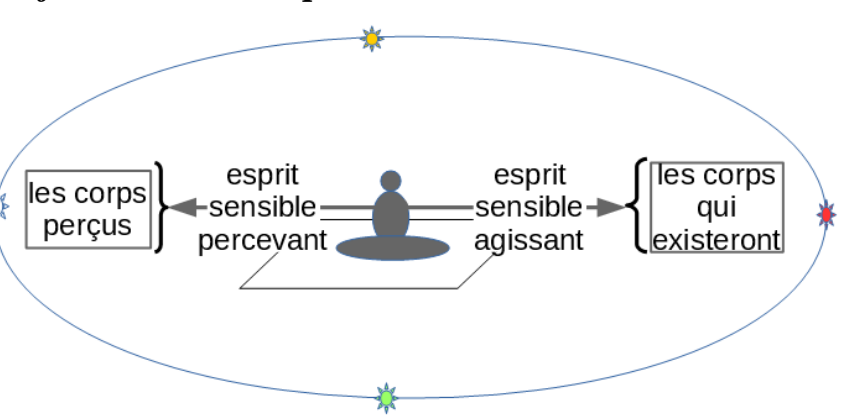

Figure 1 : les relations de l'esprit sensible, identifié au corps, Avec les corps de l'univers spatiotemporel, passé et futur situera dans le futur.

Nous avons représenté par des couleurs les "différents" soleil que l'humain perçoit au cours de la journée du fait de la circulation apparente du soleil autour de l'humain. C'est cette circulation qui impose son temps au corps de l'humain. Dans la figure 1, le soleil du lever du jour est "blanc". Au zénith il est "jaune". Le soir il est "rouge" et au nadir il est "vert."

\subsubsection{Le fonctionnement temporel du corps}

\subsubsection{La mise en évidence de la causalité temporelle des corps}

La biologie, en accord avec la connaissance empirique de l'acupuncture, met en évidence que le corps humain, comme les corps des animaux et des plantes, est sensible à l'influence du "temps qui passe". Le vieillissement des corps est la conséquence de l'influence des cycles des heures, des jours et des années. Les corps s'adaptent aux cycles temporels, ce qui leur permet de lutter contre ce vieillissement.

\subsubsection{La représentation systémique de l'humain charnel}

Naturellement, comme l'être humain représenté sur la figure 1, nous sommes identifiés à nos corps qui sont sur la terre. C'est-à-dire que nous ne faisons pas de différence entre notre corps physique et la conscience que nous en avons à chaque instant. Au Moyen Age nous croyons que la terre était plate et que notre corps naissait de la terre mère (génitrice) et du ciel père (géniteur). Après la révolution de Copernic et après la mise en évidence de la gravité par Newton nous savons que la terre et ronde et que notre corps est soumis à la gravitation. Cependant, la terre reste la mère et la mort de nos corps, donc notre mère et notre mort puisque, à ce stade d'évolution, nous sommes identifiés à nos corps. Puis Einstein a postulé que la gravité était contrebalancée par une mystérieuse force qui viendrait de la nature même du cosmos. Il y aurait donc deux constantes essentielles, celle de la gravité qui attire notre corps vers la terre et celle du cosmos (constante cosmologique) qui justifierait le tropisme ascensionnel des arbres et des humains.

Ce très bref rappel permet de comprendre la représentation traditionnel de l'être humain, d'avant la relativité et la psychanalyse qui sont apparues ensemble en 1905. L'être humain pourrait être représenté par un ternaire corps charnel, âme affective et esprit libre du corps et pouvant aspirer à s'élever vers le ciel.

La systémique traditionnelle n'est pas en opposition avec cette représentation. Le corps humain serait une structure "ouverte", ou plus exactement gérée par son moi conscient et pensant. A partir des 
mémoires qui sont stockées dans son inconscient le moi gère les flux entrants dans son corps et sortants de celui-ci.

\subsubsection{La représentation systémique des interrelations entre les êtres humains charnels}

Les êtres humains seraient en relation entre eux ainsi qu'avec d'autres systèmes, animaux, plantes, etc. La figure 2 représente les interrelations entre un être humain que je nomme $A$ et un autre système qui peut être un humain, un animal, une plante, etc. que je nomme B. Cette figure met en évidence que l'être humain $\mathrm{A}$ ainsi que le système $\mathrm{B}$ avec qui $\mathrm{A}$ est en relation constituent un nouveau système qui, comme tous les systèmes, fonctionne d'une façon rétroactive. En effet les moi de $A$ et $B$ gèrent leur échange de flux d'une façon rétroactive.

Le moi de A ainsi que celui de B sont identifiés aux corps qu'ils gèrent qui, pour eux, sont situés entre la terre qui leur a donné naissance (et qui les absorbera au moment de leur mort corporelle) et le ciel qui les attire vers le haut par un tropisme ascensionnel. Les mutations du ciel, représentées par les modifications du soleil et de la lune au cours de la journée, du mois et de l'année, (fig 1), ainsi que les mutations de la

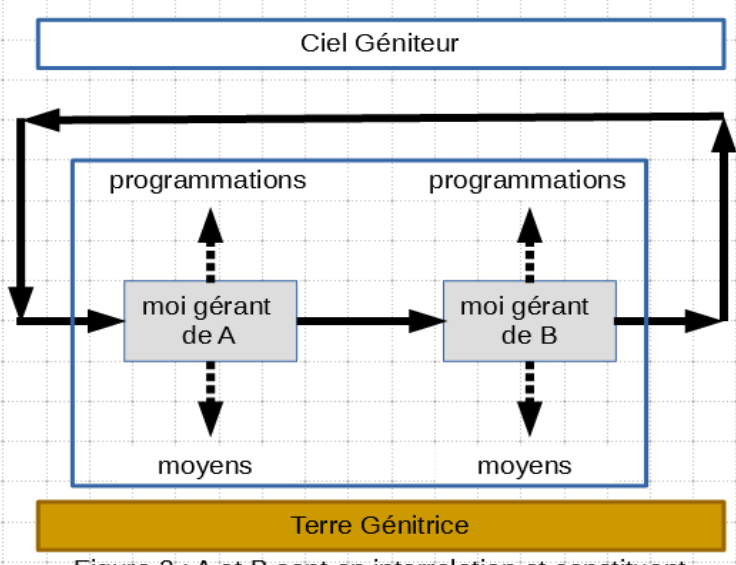

Figure 2 : A et B sont en interrelation et constituent un nouveau système.

A et $B$ sont gérés par leur moi respectifs

Les flèches pleines représentent les flux entre A et B Les flèches en pointillé représentent les buts et moyens de $A$ et $B$

terre (pluie, chaleur, vent, etc.) influent sur le fonctionnement de $\mathrm{A}$ et de $\mathrm{B}^{1}$. Symboliquement, pour les êtres humains charnels, la terre est leur génitrice et le ciel est leur géniteur.

Cette représentation systémique de l'être humain de chair, aussi importante soi-elle, ne permet pas de donner une réponse satisfaisante au paradoxe de l'invariance de la vitesse de la lumière. Ce paradoxe ne peut être compris qu'à partir de la conscience et de la relativité cognitive et systémique (RCS).

\section{CHAPITRE 2.}

\section{LA REPRÉSENTATION DE L'HUMAIN IDENTIFIE A SA CONSCIENCE}

\subsection{Mise en évidence du fonctionnement inspatiotemporel de la conscience}

Contrairement au corps, notre conscience n'est pas soumise au temps puisque nous avons conscience d'être le même, à chaque instant présent, alors que notre corps se transforme, et cela depuis notre naissance et jusqu'à notre mort. Ce fonctionnement de la conscience n'a pas été pris en compte par les scientifiques qui se présentent comme "réalistes".

Par contre certains scientifiques, dans la lignée de Louis de Broglie, ont considéré que la conscience était indispensable pour comprendre la mécanique quantique. C'est ainsi que la théorie de de BroglieBohm fait l'hypothèse que chaque objet faisant partie d'un système est associée à une onde pilote qui fonctionnerait comme la conscience humaine. Ce serait une proto-conscience qui permettrait aux

\footnotetext{
1 Le Yi King, le livre des mutations met bien en évidence cette relation masculin féminin qui existe dans l'inconscient humain pour caractériser le ciel et la terre. Le philosophe et scientifique allemand Leibniz (1646-1716) a entretenu de 1697 à 1703 une correspondance importante avec le jésuite français Joachim Bouvet (1656-1730) l'un des 5 «mathématiciens du Roi » envoyés en Chine par Louis XIV. Cela lui a permis de mettre en évidence l'importance mathématique du Yi King qui s'appuie sur les mutations du ciel et de la terre. De son côté le psychanalyste Jung a écrit, dans sa préface à la traduction du Yi King par Richard Wilhelm : «Tandis que l'esprit occidental trie, pèse, choisit, classe, isole avec soin, le tableau chinois du moment embrasse tout, jusqu'au détail le plus mince et le plus dépourvu de sens, parce que le moment observé est fait de tous les ingrédients.»

Aussi le geste simple qui consiste à interroger l'oracle du Yi King à l'aide de trois pièces de monnaie (ou de quarante-neuf baguettes d'achillée selon la tradition) fait partie du moment observé, et de ce fait répond à l'interrogation par une coïncidence toute chargée de sens. Ici on entre dans le domaine de la synchronicité, concept qui s'oppose au strict point de vue causal... Avec la synchronicité nous entrons dans le domaine de la systémique !
} 
objets de "savoir" ce qu'ils doivent faire... Ce serait donc elle qui serait à l'origine de la vie. Ce serait elle qui permettrait de comprendre les paradoxes temporels de la mécanique quantique.

La RCS est dans la lignée de ces penseurs non-réalistes

Dans le cas de l'être humain de la figure 1, ce serait sa conscience qui lui permettrait de savoir que les soleils du matin, du zénith, du couchant et du nadir, correspondent aux mutations d'un même soleil qui aurait une identité intemporelle ou éternelle.

Cela pose la question de la définition de l'identité inspatiotemporelle d'un être que nous percevons par nos sens. C'est ce que nous allons voir maintenant.

\subsection{Définition de l'être humain "conscient"}

Pour que l'exposé soit facilement partageable, l'être humain A s'exprime à la première personne en disant : Moi A je suis physiquement le même que le charnel représenté par la figure 1. Par contre j'ai une pleine conscience que c'est le même soleil que je perçois à toutes les heures du jour. Cette conscience met fin a mon fonctionnement machinal où j'étais inconsciemment soumis au temps qui passe. Ce fonctionnement "cognitif", me permet de comprendre intellectuellement que le soleil a une identité absolue, ou réelle, qui ne dépend pas de mes perceptions sensibles. Je comprends aussi que l'identité du soleil ne peut pas être définie indépendamment de moi, l'humain qui l'observe et qui le nomme.

Je peux exprimer l'identité relationnelle du soleil par : "moi A, j'ai conscience que le soleil existe". Cette formulation fait apparaître ma conscience comme étant "ce" qui me relie à chaque instant, en tant que conscience gérante de mon corps, à la conscience du gérant d'un autre système qui peut être un humain, une plante, le soleil, etc.

Plus profondément j'ai conscience que l'essence du soleil est son l'existence qui ne dépend ni du temps ni de l'espace. Je peux dire que l'essence du soleil est inspatiotemporelle. C'est-à-dire qu'à un niveau profond ce sont nos essences, à moi et au soleil, qui sont reliés hors du temps et de l'espace d'une façon inspatiotemporelle.

Ceci est vrai pour tous les systèmes de l'univers. Ce que j'exprime en disant: "l'essence de tous les êtres est leur Existence". Donc je peux exprimer mon essence en disant "Moi, j'ai Conscience d'Exister". Je formule cette Conscience Absolue d'Être par JeSuis (en un seul mot et avec des majuscules).

JeSuis est donc la source absolue et mystérieuse de ma conscience d'être un être humain nommé A. Cette Conscience d'Être peut être comprise intellectuellement mais elle ne peut être vécue que lorsque moi A j'entre dans un état de non-pensée absolue que les mystiques de toutes les religions connaissent. Les bouddhistes et les chrétiens ${ }^{2}$ donnent le

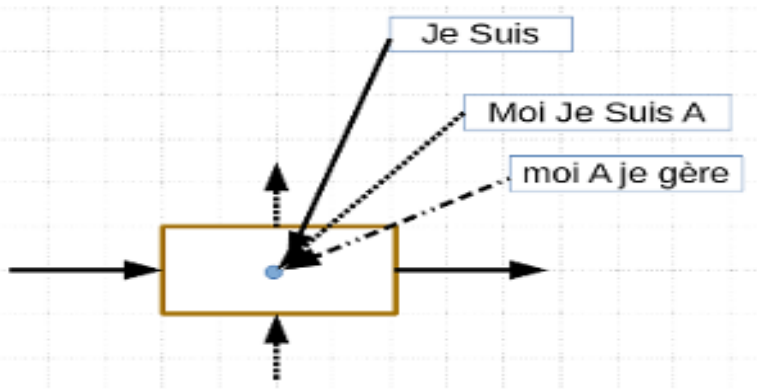

Figure 3 : L'être humain conscient JeSuis le Pilote universel

"Moi Je Suis" A le pilote individuel du corps de A "moi je suis" le gérant du corps de A nom de vacuité à cet état de conscience.

Dans cet état de vacuité j'ai une Conscience Absolue d'Être qui s'exprime au plus profond de ma conscience en disant "JeSuis, J'Existe".

Simultanément, au plus profond de ma conscience, j'ai conscience du Non-Être, le Néant cognitif qui est la vacuité. J'ai alors conscience d'être un individu, une Conscience Absolue d'Être Moi située entre JeSuis et le Néant. Symboliquement Moi Je Suis enfant de JeSuis et du Néant qui constituent le couple des géniteurs de ma Conscience Absolue d'être Moi, éternellement à l'instant présent.

Mon Moi Absolu est donc défini par : "Moi Je Suis entre l'Être et le Non-Être que je relie" ; ou :

"Moi JeSuis l'Être et le Non-Être de A".

2 Confer les enseignements de maître Eckhart. 
Ce Moi Absolu n'est pas le gérant de mon corps. Il en est le pilote qui, à chaque instant, lui donne la vie ou qui peut mettre fin à celle-ci.

Le gérant de mon corps physique est ma conscience d'être moi, un être humain nommé A.

Mon moi gère mon corps physique en étant en relation de conscience avec d'autres êtres ou systèmes de l'univers.

Mon moi est donc la conséquence de l'identité éternelle de mon Moi qui naît de JeSuis et du Néant.

Par définition un tel être humain qui définit son identité par sa conscience et non par son corps est un être "conscient".

La figure 3 représente un être humain "conscient".

\subsection{La transcendance du Pilote et du Moi}

JeSuis est donc la Conscience du Pilote Universel présent au cœur de tous les systèmes de l'univers. JeSuis transcende leur identité physique. Symboliquement JeSuis est le Géniteur ou Père des Consciences des Moi des pilotes de tous les systèmes de l'univers qui, tous, gèrent leurs corps, et leurs relations avec d'autres systèmes, par leur moi analogue à la personne humaine.

Dans chaque système, la RCS met en évidence que le "Moi JeSuis l'Être et le non-Être" est l'Observateur Relativiste (OR) qui est émetteur et récepteur d'une (onde de) conscience qui correspond à l'onde pilote de la théorie de De Broglie - Bohm. Cette onde de conscience relie d'une façon inspatiotemporelle les consciences des pilotes de tous les systèmes de l'univers. Donc tous les pilotes des systèmes de l'univers constituent un réseau qui, à chaque instant, transcende les relations entre les corps des systèmes. Ce serait ce réseau intemporel qui permettrait la vie spatiotemporelle de l'univers.

JeSuis, le Pilote Universel mis en évidence par la RCS, est créateur de la conscience du Moi éternel de chaque système par l'intermédiaire de "JeSuis l'Être et le Non-Être" qui est sa Parole, son Verbe créateur. C'est l'Esprit du Verbe qui crée le Moi de chaque être ou système. Il est donc possible de rapprocher le ternaire "JeSuis, le Verbe, l'Esprit du Verbe" du Dieu trinitaire de la religion chrétienne.

Enfin, nous avons déjà signalé que le Néant, Génitrice des Consciences des Moi des pilotes de tous les systèmes, correspond à la vacuité du bouddhisme.

Ces réflexions cognitives ont des conséquences sociales importantes.

\subsection{Conséquence sociale de cette définition systémique de la conscience}

La définition systémique des consciences comme étant "ce" qui relie les gérants des systèmes entre eux, ET qui relie les pilotes des systèmes entre eux, est une formulation anthropomorphique. Elle présente l'énorme avantage de rapprocher les religions monothéistes occidentales, et en particulier la religion chrétienne, et toutes les composantes mystiques des religions, en particulier le bouddhisme oriental. Cela peut donc être un facteur de paix essentiel au niveau de la planète.

En effet, la religion chrétienne postule l'existence de Dieu au cœur des consciences de tous les êtres humains comme l'exprime le texte du concile Vatican $\mathrm{II}^{3}$ donné en pj. Cette formulation est conforme au fait que Dieu peut être identifié avec le Pilote Universel qui est la Conscience d'Être, Père de toutes les consciences.

De son côté, le bouddhisme postule que les corps sont soumis à l'impermanence qui est la causalité temporelle. Pour le bouddhisme tous les corps naissent à l'instant présent de la vacuité par la relation de conscience entre les gérants des systèmes de l'univers. Mais les bouddhistes ignorent l'existence du Pilote Universel JeSuis en nous comme au cœur de tous les systèmes. C'est ce que la relativité

\footnotetext{
$3 \mathrm{Cf}$ le texte du concile Vatican 2. "Au fond de sa conscience, l'homme découvre la présence d'une loi qu'il ne s'est pas donnée lui même, mais à laquelle il est tenu d'obéir. Cette voix, qui ne cesse de la presser d'aimer et d'accomplir le bien et d'éviter le mal, au moment opportun résonne dans l'intimité de son cœur : "fais ceci, évite cela ». Car c'est une loi inscrite par Dieu au cour de l'homme, sa dignité est de lui obéir, et c'est elle qu le jugera. La conscience est le centre le plus secret de l'homme, le sanctuaire où il est seul avec Dieu et où sa voix se fait entendre".
} 
cognitive et systémique (RCS) vient corriger en montrant que le Pilote Universel est l'Observateur Relativiste.

Dans ces conditions il est possible de rapprocher le Christ éternel et non-né du Bouddha qui serait l'aboutissement du chemin d'éveil de la conscience de la personne humaine, gérante de son corps physique.

Le Moi, enfant de JeSuis Père et du Néant Mère, serait le Christ éternel et non né révélé par Jésus. Il se manifesterait en tant que la personne humaine, le moi de la psychologie, gérante du corps.

Le Bouddha serait l'aboutissement du chemin de libération de la conscience du moi, la personne humaine lorsqu'elle s'est libérée de tous ses affects perturbateurs. L'hindouisme le nomme le Soi. Or le dernier affect qui nous perturbe tous est l'identification inconsciente de notre esprit pensant à la causalité temporelle. Or nous avons montré que notre conscience qui naît de la Conscience d'Être et du Non-Être est éternellement libre du temps. Le Bouddha Primordial, racine de tous les Bouddha, est donc le Christ éternel et non né. Il peut naître aujourd'hui, à l'aube du $\mathrm{III}^{\circ}$ millénaire.

Je précise que les deux religions chrétienne et bouddhiste n'ont pas le monopole de l'introspection mystique. Toutes les religions, même si elles l'ignorent, essayent de codifier ce qu'elles ont compris de la conscience. Toutes essayent de formuler leurs compréhensions par des dogmes.

Nous constatons donc que la RCS permet de rapprocher dans un savoir commun la science profane de toutes les spiritualités.

Elle peut donc contribuer à rapprocher tous les êtres humains dans une même connaissance de la conscience qui transcende notre vision purement sensible de notre environnement. C'est l'évolution de l'humanité qu'avait envisagé Teilhard de Chardin lorsqu'il dit que nous passons de la biosphère, celle des humains identifiés à leurs corps biologiques, à la noosphère, celle des humains identifiés à leurs consciences et à leurs esprits.

\subsection{La représentation systémique du fonctionnement d'un être humain "conscient"}

Nous pouvons maintenant représenter le fonctionnement relationnel de deux êtres humains A et $\mathrm{B}$ conscients l'un et l'autre, ou d'un être A avec un système $B$ qui peut être extérieur à son corps (animal, plante, étoile, etc.) ou intérieur à son corps (organe, cellule, atome, etc).

La figure 4 montre deux consciences d'êtres humains $\mathrm{A}$ et $\mathrm{B}$ qui échangent des informations sensibles par leurs sens (flèches pleines). Ces informations sont gérées par leur moi (Cf fig 2). Elles ne sont possibles que parce que A et B sont intriqués (flèches en pointillé) au niveau de leurs Moi.

L'intrication s'effectue au niveau des Moi, les

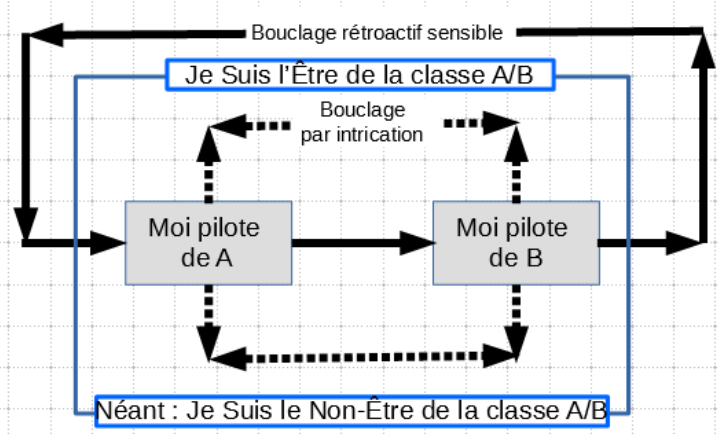

Figure 4 : A et $\mathrm{B}$ sont reliés par intrication intemporelle car $A$ et $B$ ont le même but - flèches en pointillé Cela définit une classe d'êtres.

C'est l'intrication qui permet la relation sensible des flux entre A et B (flèches pleines)

Consciences pilotes de $\mathrm{A}$ et $\mathrm{B}$, qui ont conscience l'une et l'autre de naître de JeSuis à l'instant présent. Cela crée un réseau de systèmes $\mathrm{A}, \mathrm{B}, \mathrm{C}$, etc. qui constituent une classe d'êtres qui est définie par nos Moi qui parlent en nous en disant: "Moi A JeSuis l'Être et le Non Être du pilote de A". L'exemple le plus évident de classe d'êtres fonctionnant en réseau est celui des êtres humains de la terre. Les animaux et les plantes fonctionnent eux aussi en réseau.

La classe des humains est définie par l'Homme archétypal qui est la conscience inspatiotemporelle du Moi naissant du Verbe ("JeSuis la Conscience d'Être et de Non-Être") habitant un corps physique soumis au temps. Ce dernier étant géré par le moi, la personne humaine.

Les humains A, B, C, du réseau (la classe) des êtres humains du passé, du présent et du futur, peuvent se regrouper pour former des systèmes qui sont gérés et pilotés. Ces systèmes ont donc un gérant et une loi. Leur identité est définie de la façon suivante : Ils (les membres de ces systèmes) reconnaissent 
que leurs Moi naissent du Pilote universel (JeSuis, le Verbe, l'Esprit du Verbe). C'est le cas des familles humaines lorsqu'elles se définissent par leur soumission au Pilote Universel.

Les membres d'une même classe se reconnaissent et peuvent avoir des échanges.

La Conscience du Moi est exclusive. C'est-à-dire que le Néant est le fondement de toutes les classes de conscience des êtres. La mort de la conscience correspond à une disparition pure et simple de la conscience.

En même temps, les classes des consciences des (Moi des) êtres humains sont inclusives. C'est-à-dire que A peut appartenir à une famille de sang par ses parents et à une communauté particulière, qui est sa famille spirituelle (dans le sens de communauté d'esprit) qui appartient à l'humanité de la terre. Sa conscience (celle de son Moi) peut aussi appartenir à une conscience cosmique, celle du système solaire, puis celle de la galaxie et enfin celle de l'univers.

Cette inclusion des classes permet de comprendre que tous les êtres de l'univers constitue une classe universelle. Tous les êtres de l'univers se reconnaissent et peuvent s'unir pour agir ensemble car ils sont tous constitués par le même composant de base qui est le vion, analogue au bit de l'informatique ${ }^{4}$.

Nous commençons seulement à prendre conscience de cette interdépendance de tous les êtres qui a été mise en évidence dans le cas des arbres par Peter Wohlleben ${ }^{5}$

\section{6. la naissance de l'humanité nouvelle du III $^{\circ}$ millénaire}

L'être humain de la terre peut être considéré comme étant l'aboutissement provisoire de l'évolution de la terre.

Cette évolution qui a duré 4 milliards d'années s'est manifestée dès le début de l'univers il y a 13,7 milliards d'années, par des regroupements de vions en systèmes de plus en plus complexes. Cette évolution étant pilotée par le Pilote ternaire [JeSuis, le Verbe, l'Esprit du Verbe] qui serait présent au cœur de tout système en tant que fondement du Moi du pilote du système considéré. Ce Moi étant l'OR mis en évidence par la relativité d'Einstein, puis par la RCS.

La compréhension de la RCS comme fondement possible de la science systémique de l'esprit et de la conscience, permet de comprendre que le stade actuel de l'évolution est celui de la naissance de la noosphère annoncée par Teilhard de Chardin.

Nous assistons à la naissance de l'humanité dans laquelle les états, aussi puissants soient-ils, ne peuvent plus agir d'une façon solitaire. Les USA, l'Europe, la Russie, la Chine, l'Inde, l'Amérique du sud, l'Afrique, l'Australie, ont des échanges économiques sous-tendus par l'informatique.

En même temps les religions ne peuvent plus régner sur des territoires à elles car les peuples sont mélangés.

Nous assistons donc à l'émergence d'un ordre mondial. Nous n'en sommes pas encore à un gouvernement mondial mais nous nous dirigeons peut être vers une gestion fédérée de la terre comme se sont dotés les états unis d'Amérique.

En même temps les religions traditionnelles qui sont nées au cours du $\mathrm{I}^{\circ}$ millénaire, ou même avant pour l'hindouisme, le bouddhisme, le taoïsme et le chamanisme, doivent apprendre à cohabiter. Là encore nous n'en sommes pas encore à une religion mondiale de l'Homme Conscient, mais des signes très positifs de dialogues interreligieux sont visibles.

C'est-à-dire qu'au plan économique, politique ou religieux, l'être humain en tant que tel, et non plus en tant que membre d'une communauté particulière, fait son apparition. Il n'a pas encore d'identité reconnue mais il est déjà là.

L'intérêt de la systémique cognitive qui s'appuie sur la RCS est de pouvoir définir cet être humain "nouveau" qui est une conscience habitant et gérant un corps et non plus un corps pensant.

Sur le plan religieux, nous avons déjà signalé que le concile Vatican 2 a défini Dieu par sa présence dans la conscience humaine. Il est nécessaire de faire un pas de plus et de définir l'être humain par sa conscience. Dieu devenant sa Conscience Primordiale d'Être.

$4 \mathrm{Cf}$ : la relativité cognitive et systémique - définition du vion

5 La vie secrète des arbres par Peter Wohlleben - éditions Les Arènes 
A ce moment-là, la science des systèmes cognitifs deviendra la nouvelle science qui prendra la suite de la science "réaliste" des objets.

\section{CONCLUSION}

La crise actuelle que traverse l'humanité doit être considérée comme étant un changement radical de paradigme qui concerne tous les domaines des activités humaines, aussi bien scientifiques que philosophiques ou religieux.

C'est véritablement une nouvelle humanité qui est en train de naître. Nous tous, hommes et femme de la terres, nous sommes concernés par cette naissance puisque notre corps est la génitrice de notre nouvelle conscience d'être humain, notre père étant JeSuis, la Conscience Absolue d'Être qui est au cœur de notre conscience et au cœur de tous les composants de notre corps physique.

L'"enfant" qui naît est notre nouvelle conscience, libre du temps et de l'espace, et pourtant totalement responsables individuellement et collectivement du devenir de la terre et du cosmos.

Cette naissance de "l'enfant nouveau" qui devient membre de l'univers peut être considérée comme étant la véritable guérison de l'être humain.

\section{BIBLIOGRAPHIE}

BOHM, David (1989). La Plénitude de l'univers. [«Wholeness and the Implicate Order »]. Éditions du Rocher.

BOHM, David \& KRISHNAMURTI, Jiddu (1989). Le temps aboli : Dialogues. [« The Ending of Time »]. Éditions du Rocher, 1989.

BOHM, David \& PEAT, David (1990). La Conscience et l'Univers. [«Science, Order, and Creativity »]. Éditions du Rocher.

BOHM, David (1997). Pour une révolution de la conscience [« Changing Consciousness: Exploring the Hidden Source of the Social, Political and Environmental Crises Facing our World »], Éditions du Rocher.

BROGLIE, Louis (de). Annales de la fondation louis de Broglie.

CHARON, Jean (1977). Théorie de la relativité complexe. Albin Michel.

Concile Vatican 2 (Gaudium et Spes, ${ }^{\circ} 16$ ).

EBO, Romual. Expérience spirituelle et conscience chez John Henry Newman. Editions Saint Leger.

TEILHARD DE CHARDIN, Pierre. Le phénomène humain. L'avenir de l'Homme; La place de l'Homme dans l'univers.

VITRAC, Richard (2011). "Interprétation systémique du paradoxe de la lumière". Acta Europeana Systemica (AES), $\mathrm{n}^{\circ} 1$.

VITRAC, Richard (2017). "La théorie de la relativité cognitive". Acta Europeana Systemica (AES), $\mathrm{n}^{\circ} 7$.

VITRAC, Richard (2019). L'école de la conscience. The bookEdition.

VITRAC, Richard (2018). "La démonstration systémique des formules de Lorentz". Acta Europeana Systemica (AES), n8.

LUYCKX-GHISI, Marc, VITRAC, Maxime, VITRAC, Richard (2018). "L'Europe au cœur de la crise de l'humanité : Une approche systémique de la santé de l'Europe à partir de la relativité cognitive et systémique". Acta Europeana Systemica (AES), n8.

VITRAC, Richard (2017). Mon nom est Je Suis. Edilivre.

VITRAC, Richard (2016). Le grand architecte de l'univers : Réponse à Stephen Hawking. Editions EUE. 